\title{
La colectividad como resistencia en la cuenca alta del río Chicamocha*
}

COLLECTIVITY AS RESISTANCE IN THE UPPER BASIN OF THE CHICAMOCHA RIVER

A COLETIVIDADE COMO RESISTÊNCIA NA BACIA SUPERIOR DO RIO CHICAMOCHA

\section{Laura López Estupiñán**}

Cuadernos de Música, Artes Visuales y Artes Escénicas

/ Volumen 14 - Número 2 / Julio - Diciembre de 2019

/ ISSN 1794-6670 / Bogotá, D.C., Colombia / pp. 43-53

Fecha de recepción: 11 de diciembre de 2018

Fecha de aceptación: 19 de febrero de 2019

Disponible en línea: 24 de junio de 2019

doi 10.11144/javeriana.mavae14-2.Iccr

* Artículo de reflexión. Hace parte del proceso colectivo de investigación, defensa y protección del patrimonio cultural en Boyacá.

* Antropóloga por la Universidad del Cauca y magíster en Arqueología Sudamericana por la Université de Rennes 1 y la Universidad Nacional de Trujillo. Docente de la Escuela de Ciencias Sociales de la Universidad Pedagógica y Tecnológica de Colombia, directora del Museo Arqueológico de Tunja e investigadora del Grupo Interdisciplinario de Investigaciones Arqueológicas e Históricas de la misma universidad. ORCID 0000-0002-9923-3984

\section{Cómo citar:}

López Estupiñán, Laura. 2019. “La colectividad como resistencia en la cuenca alta del río Chicamocha". Cuadernos de Música, Artes Visuales y Artes Escénicas 14 (2): 43-53.

http://doi.org/10.11144/javeriana.mavae14-2.Iccr 


\section{Resumen}

Sistematizar las experiencias colectivas y generar reflexiones en torno a la resistencia de comunidades locales frente a los proyectos extractivos en Boyacá es el aporte de este artículo. Se recurre a las experiencias colectivas exitosas que han favorecido la defensa y la protección del agua en la cuenca alta del río Chicamocha de 2010 a 2018, principalmente en la provincia de Sugamuxi, en Colombia. Mediante un trabajo interdisciplinar de las artes, la antropología, la ecología, el derecho, las licenciaturas, las ingenierías y los saberes locales, se garantizó la revisión de los estudios de impacto ambiental y planes de manejo, la traducción de los lenguajes técnicos a cotidianos, la participación activa en los mecanismos de participación ciudadana y el acompañamiento a las comunidades afectadas por los trabajos de exploración sísmica de dos proyectos de hidrocarburos en cercanías del lago de Tota. El artículo permite repensar los conceptos clásicos de familia, comunidad y movimiento social desde la acción colectiva de protección y defensa del agua, liderada por los habitantes de la provincia de Sugamuxi, quienes estudiaron los documentos entregados por las empresas y evidenciaron las afectaciones e irregularidades en audiencias públicas y procesos de seguimiento ambiental. La dinámica permitió el reconocimiento de un territorio, la generación de metodologías propias que vincularon la comunicación alternativa, la acción política y la argumentación técnica con incidencia en el licenciamiento ambiental de dos proyectos de hidrocarburos en la cuenca alta del río Chicamocha.

Palabras clave: agua; territorio; colectivos; resistencia; conflicto.

\section{Abstract}

The purpose of this paper is the systematization of collective experiences and the generation of reflections on the resistance of local communities to extractive projects in Boyacá. It draws on the successful collective experiences that have favored the defense and protection of water in the upper Chicamocha river basin from 2010 to 2018, mainly in the province of Sugamuxi, Colombia. An interdisciplinary work between the arts, anthropology, ecology, law, different bachelor's degree and engineering programs, as well as local knowledge, guaranteed the review of environmental impact studies and management plans, the translation of technical jargon into everyday language, active participation in citizen participation mechanisms, and the support to communities affected by the seismic exploration work of two hydrocarbon projects near Lake Tota. The paper makes it possible to rethink the classic concepts of family, community and social movement from the collective action of protection and defense of water, led by the inhabitants of the Sugamuxiprovince, who studied the documents provided by the companies and evidenced the affectations and irregularities in public hearings and environmental monitoring processes. The activity enabled the recognition of a territory, the generation of own methodologies that linked alternative communication, political action and technical argumentation with incidence in the environmental licensing of two hydrocarbon projects in the upper basin of the Chicamocha river.

Keywords: Water; territory; collective;

resistance; conflict.

\section{Resumo}

A contribuição deste artigo é a sistematização das experiências coletivas e a geração de reflexões sobre a resistência das comunidades locais aos projetos extrativistas em Boyacá. Usam-se experiências coletivas bem-sucedidas que favoreceram a defesa e a proteção da água na bacia do alto rio Chicamocha de 2010 a 2018, principalmente na província de Sugamuxi, na Colômbia. Através de um trabalho interdisciplinar de artes, antropologia, ecologia, direito, licenciaturas, engenharias e conhecimento local, garantiu-se a revisão de estudos de impacto ambiental e planos de gestão, a tradução de linguagens técnicas para cotidianas, a participação ativa em mecanismos de participação cidadã e o acompanhamento das comunidades afetadas pelas obras de exploração sísmica de dois projetos de hidrocarbonetos perto do Lago de Tota. O artigo permite repensar os conceitos clássicos de família, comunidade e movimento social a partir da ação coletiva de proteção e defesa da água, liderada pelos habitantes da província de Sugamuxi, que estudaram os documentos entregues pelas empresas e evidenciaram as afetações e irregularidades nas audiências públicas e nos processos de monitoramento ambiental. A dinâmica permitiu o reconhecimento de um território, a geração de metodologias próprias que relacionaram a comunicação alternativa, a ação política e a argumentação técnica com incidência no licenciamento ambiental de dois projetos de hidrocarbonetos na parte alta da bacia do rio Chicamocha.

Palavras-chave: água; território; coletivos; resistência; conflito. 


\section{INTRODUCCIÓN}

La cuenca alta del río Chicamocha la conforman 21 municipios del departamento de Boyacá. Santa Rosa de Viterbo, Duitama, Nobsa, Tibasosa, Sogamoso, Paipa, Sotaquirá, Tuta, Firavitoba, Iza, Cuítiva, Pesca, Tota, Toca, Siachoque, Soracá, Tunja, Motavita, Cómbita, Chivatá y Oicatá son municipios que hacen parte del altiplano y 13 subcuencas, sus depresiones obedecen al plegamiento de rocas sedimentarias, elevadas por el proceso de orogenia andina que favorece la presencia de $15000000 \mathrm{~m} 3$ de agua superficial, la recarga de acuíferos, la formación de ecosistemas tropicales con baja precipitación anual y picos hasta de 4000 msnm (Corpoboyacá 2006), condiciones que favorecieron la ubicación de asentamientos a lo largo y ancho de la cuenca desde épocas prehispánicas que corresponden a cazadores-recolectores (Rodríguez 2003), herreras, muiscas, coloniales y contemporáneos.

Por estudios arqueológicos sabemos que las condiciones ambientales y geográficas han permitido la permanencia de pobladores por más de 10000 años en este territorio, quienes cultivaban alimento y extraían minerales, por lo que generaron procesos complejos de adaptación y desarrollo humano. A mediados del siglo XX llega la gran industria extractiva de hierro y con ella se fortalece la demanda de proyectos mineros, lo que aumenta la erosión, desertificación, salinidad y escasez de agua con la sobreutilización del 43 \% de los suelos (IGAC 2014).

Las dinámicas de acceso y control de la tierra están condicionadas por economías globales que fomentan el monocultivo y la industria en medio de títulos de falsa tradición que llegan al 70\% de la informalidad (Reyes 2014). El problema aumenta cuando se organizan colectivos que se oponen a los proyectos de gran industria, como la minería y los hidrocarburos, puesto que la mayoría de la población ve en ellos la posibilidad de acceder a un trabajo, mirada coincidente en el Plan Nacional de Desarrollo 2010-2014: Prosperidad para todos y el Plan Nacional de Desarrollo 2014-2018: Todos por un nuevo país.

Aunque los campesinos reconocen las afectaciones de la industria minera y de hidrocarburos, los más jóvenes recurren a los proyectos en búsqueda de dinero, por lo que queda en el campo una población geriátrica que no tiene las fuerzas para continuar labrando la tierra. Por su parte, la población adulta recurre a cualquier trabajo que genere ingresos, lo que institucionaliza la esclavitud en la agricultura del capital intensivo y aumenta los procesos erosivos de los suelos (Foster 1980).

Este panorama permite comprender la escasa participación de los habitantes en los procesos de ordenamiento territorial, su inclusión en el desarrollo de políticas y el empoderamiento de resistencias colectivas aunadas en la defensa de los derechos fundamentales. Pese a la poca participación, encontramos diversidad de colectividades dispuestas a defender el agua como elemento fundamental, las montañas como punto de referencia en el paisaje y la tranquilidad que por años se ha vivido en el territorio. Aunque las acciones no han traído los muertos que han cobrado resistencias en otras partes del país, los conflictos generados en el territorio han sido evidentes.

Los proyectos de exploración y explotación de hidrocarburos en la cuenca alta del río Chicamocha empiezan con el siglo XXI. El Proyecto MNorte fue concesionado a la empresa Hocol S. A. en 2000 y cedido desde 2010 a la empresa francesa Maurel \& Prom. Acogió un área inicial de 25294 ha, de las que el 67,46 \% estaban entre los 3000 y 3600 msnm. Inició exploración sísmica durante 2012 en los municipios de Pesca, Tota, Cuítiva, Iza y Firavitoba, a unos cuantos metros de la laguna de Tota, y en Iza, municipio declarado bien de interés cultural de la nación. 
Con negativa en el acceso a predios por parte de los propietarios, se evidenciaron las amenazas e intimidaciones por parte de funcionarios de empresas, instituciones y fuerza pública (Getulio Muisca 2012), quienes violaron la propiedad privada y autonomía de los campesinos. Como resultado de la exploración, se agrietaron casas en varios municipios y resultaron averiadas obras de infraestructura pública como el acueducto de Firavitoba (Acosta 2015; Getulio Muisca 2012a). Luego de múltiples denuncias por parte de la comunidad y el Colectivo por la Protección de la Provincia de Sugamuxi, la Procuraduría General de la Nación (2012) expide un concepto técnico para el proyecto y la Contraloría General de la República (2013) se pronuncia con el Informe de actuación especial para el manejo de la laguna de Tota.

A su vez, el Instituto Colombiano de Antropología e Historia (ICANH) ${ }^{1}$ solicita en 2014 un procedimiento de obligatorio cumplimiento por la intervención del pozo Balsa 1 sin el debido trámite arqueológico. Ante los actos irresponsables del proyecto, el Colectivo por la Protección de la Provincia de Sugamuxi recurre a la divulgación del problema en espacios de participación ciudadana y mediante redes sociales, historietas y videos, lo que incidió en la reducción del área por intervenir.

Cuatro años después de la concesión del Proyecto MNorte, se adjudica concesión al Proyecto Buenavista mediante contrato entre la Agencia Nacional de Hidrocarburos (ANH) y la empresa Omega Energy, con un área aproximada de 8036 ha. En 2007, el Ministerio de Ambiente y Desarrollo Sostenible otorga la licencia ambiental que dio vía libre al Proyecto Área de Interés para el Desarrollo del Campo Buenavista. Desde 2007 a 2013, cuando se solicita modificación de la licencia, el Ministerio de Ambiente, Vivienda y Desarrollo Territorial impuso varios sancionatorios y medidas preventivas (Colectivo por la Protección de la Provincia de Sugamuxi 2018).

Pese a los sancionatorios, Omega Energy solicita modificación de licencia ambiental en varios municipios de la cuenca. Con ocho pozos en producción durante fase exploratoria y las múltiples denuncias de habitantes en materia ambiental (disminución de caudal de fuentes hídricas, expulsión de lodos), afectaciones a casas, cultivos y fuentes de trabajo, el desplazamiento de una vereda por dos patadas de pozo en Corrales y la precariedad en vías de acceso (Getulio Muisca 2013, 2014), la Autoridad Nacional de Licencias Ambientales (ANLA) niega la modificación de licencia ambiental en 2014 (Resolución 0376/2014, de 11 de abril; Resolución 0867/2015, de 24 de julio).

Las negaciones de modificaciones ambientales y la reducción de áreas por intervenir fue posible por un proceso de documentación y participación activa en las socializaciones de los proyectos, en que se evidencian múltiples irregularidades e incoherencias técnicas. Ante la situación, inicia un proceso de reflexión y preocupación colectiva, que convoca y ejerce acciones comunes para esclarecer las consecuencias de los proyectos en el área. En medio de las reflexiones y preocupaciones, nace el Colectivo por la Protección de la Provincia de Sugamuxi, acción colectiva interesada en comprender los proyectos de exploración y explotación de hidrocarburos. Sin ser expertos en la disciplina y siguiendo las intuiciones de posibles afectaciones al territorio, se encontró que 52 bloques petroleros en Boyacá, que corresponden a más de la mitad de los bloques petroleros nacionales, se superponían con 1540 títulos mineros (Pineda 2014), lo que aumentaba la crisis ambiental, sanitaria y agrícola en el departamento (López 2015).

La preocupación aumenta con la superposición de títulos y las consecuencias de la minería en la salud de pobladores de la cuenca alta del río Chicamocha, ante el hecho se inicia un diálogo interdisciplinar articulado con reconocimientos territoriales, documentación de las afectaciones, confrontación técnica en las socializaciones y la búsqueda de expertos en la 
disciplina para que aclararan las implicaciones de los proyectos. Si bien la argumentación técnica fue necesaria, durante el proceso de protección territorial se recurrió a metodologías propias, formas de hacer intuitivas que vinculaban el arte, la comunicación, las prácticas artísticas y las dinámicas cotidianas en resistencias colectivas que no daban lugar a la duda, sino que por el contrario se adaptaron y transformaron de acuerdo con el contexto y con la situación.

Algunos de los conflictos socioambientales generados por la exploración y explotación de hidrocarburos se resumen en el quebrantamiento del tejido social (Acosta 2015) y en el desconocimiento de las dinámicas socioculturales vinculantes del patrimonio cultural de los pueblos. En cuanto al quebrantamiento del tejido social, se identificó que los empleados de las empresas recurren a engaños que alientan la firma de permisos y accesos a los predios de la gente, sin especificar las reales consecuencias de la exploración petrolera, como el incurrir "en falsedad de documento, al entregar información sobre los riesgos para la salud humana y la afectación de los recursos naturales que representa el uso de material explosivo sismigel" (Colectivo por la Protección de la Provincia de Sugamuxi 2013).

El explosivo fue usado por la empresa para detonar 18225 t en 25000 ha, lo que generó averías en potreros, casas, movimientos telúricos y afectaciones pecuarias. Dos y cuatro años después llegaron las denuncias de más de 210 pozos de agua "desaparecidos" en los municipios de Pesca, Iza, Tota, Cuítiva y Firavitoba (Contagio Radio 2016). La profundización del agua por parte de la actividad sísmica generó el desplazamiento de familias que por falta de agua en sus predios y casas fueron obligadas a abandonar sus predios, como se observa en los testimonios recopilados antes, durante y después de las audiencias públicas ambientales (Colectivo por la Protección de la Provincia de Sugamuxi 2016).

\section{METODOLOGÍAS Y RESISTENCIAS PROPIAS EN UN CONFLICTO AMBIENTAL}

Con la llegada de los proyectos extractivos, se generaron procesos de cohesión social que buscaban comprender los efectos de una industria desconocida en el territorio, la industria de hidrocarburos. En ese camino, las resistencias individuales fueron consolidando resistencias colectivas que vinculaban el espíritu femenino y sensible del territorio, hombres y mujeres entendieron que había otras formas menos agresivas, más amables, no ingenuas, que permitirían mediante argumentos evidenciar los errores en la exploración sísmica y las irregularidades en los estudios de impacto ambiental.

La acción colectiva de veeduría, seguimiento y evaluación técnica estuvo acompañada de metodologías propias, prácticas artísticas, procesos de comunicación alternativa y resistencias que argumentaron la inviabilidad técnica y social de los proyectos en los espacios de participación ciudadana, la cotidianidad y el acompañamiento de los entes de control nacional. La estrategia se metaforizó con juegos de roles que permitieron comprender las posiciones y funciones de cada actor. Invertir el papel, informarse e informar, traducir documentos y socializaciones a lenguajes cotidianos, ejemplificar con realidades y argumentar la palabra fueron los instrumentos del andar.

Durante la revisión de los estudios de impacto ambiental, los planes de manejo y la participación de socializaciones, fue evidente la restricción a los documentos y el uso de lenguajes técnicos que confundían a la gente. Para ello, el Colectivo por la Protección de la

Provincia de Sugamuxi recurrió a la creación de Getulio Montaña Laguna, Quejulia Páramo 
Flores y Cubio Montaña, personajes que traducen los alcances, las implicaciones y las consecuencias de los proyectos de hidrocarburos en un lenguaje cotidiano. Los personajes tuvieron gran acogida, evitaron la señalización de actores y visibilizaron los problemas a nivel local, regional, nacional e internacional.

Siendo conscientes de los diversos tipos de población, se recurrió a la creación de cómic e historietas que articularan el territorio, la cotidianidad y el problema petrolero mediante un diálogo de los personajes, y así se generó la creación de otros como Carmina Mar, animales y miembros de la familia Montaña. Las temáticas trabajadas en el cómic varían, algunas son dibujos en tinta negra que dan cuenta del territorio y sus afectaciones, otras son ilustraciones que acompañan textos relacionados con la sísmica, los estudios y los planes de manejo, el territorio ancestral, las amenazas de la exploración petrolera, las organizaciones sociales, la protección del territorio, el páramo, el campesinado, el paro agrario campesino realizado en 2013 y los encuentros de defensores de páramos, ambientales y sociales (Getulio 2014).

Con cientos de historietas e ilustraciones, comenzó a visibilizarse un proyecto que desconocía las vocaciones agrícolas, los procesos sociales y las formas de acceso a la tierra. Su divulgación en redes sociales, el voz a voz y la pegatina en postes públicos fueron los medios más utilizados. La eficacia fue tal que desde Francia, país de una de las multinacionales que intervinieron el suelo, ARTE Radio se comunicó para seguir el caso y socializarlo en emisoras, fanzines y festivales de cine (Colectivo por la Protección de la Provincia de Sugamuxi 2019). Así fue como la historieta se consolidó en "estrategia de comunicación pensada para la divulgación de información sobre el territorio entre la comunidad", que permitió acompañar a las poblaciones afectadas por la extracción de hidrocarburos. Sus propósitos fueron la divulgación de problemas de la comunidad, la transmisión de la investigación y la intervención técnica sobre los conflictos territoriales en un lenguaje cotidiano, fortalecer la confianza entre las organizaciones de base para lograr fines comunes y empoderar a las comunidades (Mujer Rural y Derecho a la Tierra 2018).

La presencia de pocos expertos en hidrocarburos hizo necesario el diálogo de saberes, la comprensión de tecnicismos y la generación de procesos de enseñanza-aprendizaje en la disciplina. Este hecho facilitó la apropiación de un conocimiento técnico y científico en pobladores de la cuenca, y potencializó la veeduría y el seguimiento ambiental a los proyectos extractivos mediante la "expedición sísmica" liderada por pobladores (Acosta 2015). Claves como la no perforación en pendientes mayores de $45^{\circ}$ y las distancias mínimas de las fuentes de agua para la colocación de cargas (100 m para nacederos y $30 \mathrm{~m}$ para corrientes y cuerpos de agua superficiales; Ministerio de Ambiente 1997), entre otras, fueron fundamentales a la hora de registrar fotográficamente las inconsistencias de los proyectos de hidrocarburos. Con el registro fotográfico, se dio paso al reconocimiento territorial por parte de los pobladores, quienes realizaron caminatas en búsqueda de los nacimientos, cuerpos de agua superficiales y las pendientes mayores de $45^{\circ}$.

Durante el proceso se documentó la "retractación de aproximadamente 1000 permisos mediante peticiones concretas y debidamente probadas, dirigidas a las diferentes entidades estatales demostrando la flagrante violación de la Guía Sísmica, el territorio y la propiedad privada" (Acosta 2015). El ejercicio dio paso a un inventario participativo que permitió el mapeo del territorio en términos acuíferos, en el que se encontraron pozos sembrados, reservorios, caudales, nacimientos, aljibes y ojos de agua. Esta situación evidenció otras relaciones de los campesinos con el agua, que no fueron registradas por las empresas, pero sí por investigadores de la zona. 
El reconocimiento territorial también potencializó el trabajo colectivo y permitió la consolidación de redes de parentesco, amigos y vecinos que se unieron para reflexionar sobre el problema y pensar acciones que minimizarán los riesgos de la sísmica. Así fue como se recurrió a la recolección de cables en los predios no autorizados y a la celebración de vigilias ecuménicas, peregrinaciones y romerías que permitieran poner el tema en discusión colectiva entre 2013 y 2014. Conscientes de las dificultades en la comprensión del problema por parte de la población general, así como de la continuidad de las actividades sísmicas y de exploración de hidrocarburos, las acciones colectivas se tomaron los espacios de participación ciudadana, tales como audiencias públicas de seguimiento ambiental en el Congreso de la República en 2012 y audiencias ambientales en los municipios de Sogamoso en 2013 y de Pesca en 2016.

La preparación de las audiencias implicó la articulación y el trabajo en red con los afectados, quienes apropiaron la evidencia y el argumento, documentaron cada una de las afectaciones con registros fotográficos y videos, expusieron las consecuencias de los proyectos en el territorio y elevaron una voz unísona que decía: “En Boyacá no cambiamos agua por petróleo". Además de las audiencias públicas en territorio, se participó de la audiencia de seguimiento ambiental en el Congreso de la República en 2012. Con un contraste de cartografías superpuestas entre la información suministrada por las empresas, los hallazgos registrados por el Colectivo por la Protección de la Provincia de Sugamuxi y las investigaciones en el área, se garantizó una semana de sintonía en la televisión nacional sin represión y la posterior disminución de las áreas por solicitar en las modificaciones de licencias ambientales.

La alta participación ciudadana en las audiencias, la calidad de las exposiciones en términos técnicos e investigativos, los argumentos y las experiencias evidenciaron la inconformidad de las poblaciones ante las autoridades ambientales, de modo que fueron titulares de radio y prensa nacional (Caracol Radio 2016; RCN Radio 2013; Semana Sostenible 2016). El ejercicio cartográfico como escenario de reconocimiento territorial y argumento técnico estuvo acompañado de un registro sistemático audiovisual, herramienta de denuncia que documentó el desplazamiento de 180 familias en el municipio de Corrales (Getulio Muisca 2013) y la profundización de aguas en el municipio de Pesca (Colectivo por la Protección de la Provincia de Sugamuxi 2016).

El ejercicio de denuncia en redes sociales, plazas de mercado y la argumentación técnica fue importante. Durante el proceso se generaron escuelas de formación que vincularon reconocimientos territoriales, la apropiación de los lenguajes técnicos y disciplinares mediante pintura mural, talleres, juegos y exposiciones fotográficas. Para ello, se recurrió al apoyo de otros colectivos y organizaciones que apoyaran económicamente el desarrollo de dos foros de hidrocarburos en 2012 y 2013, seis escuelas rurales ambientales en 2014 y 2015, y varios talleres de capacitación en la recolección, la sistematización y el manejo de información relacionada con las afectaciones de las actividades extractivas en 2017.

Lo anterior permitió empoderar a las comunidades mediante diálogos intergeneracionales que facilitaron el reconocimiento de los pobladores como investigadores. En un principio, los adultos lideraron el proceso, pero durante el trabajo de campo se dieron cuenta de la necesidad de recurrir a sus hijos para el manejo de grabadoras, celulares, cámaras y computadores. El trabajo en familia complementó el proceso de formación y socialización del problema; como investigadores dieron cuenta de afectaciones históricas que habían causado muertes y daños irreparables al ecosistema, lo que permitió argumentar con mayor 
precisión el problema. En voz de don Pedro, "los talleres nos dieron más argumentos a la hora de dar la pelea con las empresas, nosotros sabíamos todos los daños que hacían por acá, pero no teníamos las fechas, los lugares y los dolientes" (comunicación personal, 7 de mayo de 2017).

Así fue como la experiencia individual fortaleció la acción colectiva. Sin tener claro los principios de la educación popular, sin ser expertos en docencia, iniciamos un proceso formativo interdisciplinar desde las intuiciones y los aprendizajes. En el proceso individual de protección territorial, cada uno había aprendido a escuchar el cuerpo y el territorio, a levantar la voz y a confrontar las socializaciones convocadas por las empresas cuando se decía algo impreciso, confuso y que no obedecía a la realidad territorial. Desde la sensibilidad, se recurrió a la pintura mural, lo que permitió contrastar los territorios, mostrar su belleza sin plataformas y su futuro con ellas, reivindicar lo que nos hace parte de un paisaje y compartir las preocupaciones ambientales con la población en general.

El ejercicio hizo posible comprender que lo técnico podía ser divertido, era la excusa para reunirse, encontrarse y pensar estrategias que facilitaran la lucha. Se pensó, diseñó y crearon los zurrones pa juegar, una serie de juegos que invitan a discutir y reflexionar sobre la vida, el agua, la tranquilidad, los alimentos y la palabra. Desde los encuentros, los procesos, los sueños, las ideas, los proyectos y las alternativas, se forjaron resistencias en la cuenca alta del río Chicamocha, territorio privilegiado con paisajes altoandinos, con ecosistemas que favorecen la producción de todo tipo de alimento, con gentes que mantienen relaciones culturales andinas con el lugar donde viven.

La acción colectiva asintió el sentir de los habitantes en cada uno de sus territorios, lo que permitió denunciar los impactos y, a su vez, establecer estrategias de acción inmediata. Pensar lo relatado es increíble, más aún en un país como Colombia donde las desapariciones forzadas y la estrategia del terror ha reinado históricamente; por eso, este artículo es un medio para generar esperanza en otras formas de lucha. Las marchas y protestas sociales no son los únicos medios: las acciones tienen sus propios tiempos y no hay receta para su desarrollo.

Se hace necesario reconocer la técnica del extrañamiento de la antropología, puesto que la mayor parte de las colectividades están conformadas por mujeres y jóvenes que habían estudiado y trabajado en otras ciudades, situación que había permitido dimensionar el problema. Los espejos y las realidades de los departamentos del Putumayo, de Norte de Santander y de Arauca fueron fundamentales para no aplazar la acción. La falta de agua y alimento, la erosión del suelo, la muerte de chigüiros y los sobrecostos de enseres, arriendos y predios en zonas extractivas fueron algunos de los factores que fundamentaban la no aceptación de la industria petrolera. Entre saberes y experiencias, se sumaron las inquietudes, las dudas, los reclamos y las inconsistencias con profesionales en el sector de hidrocarburos.

Mantener la acción colectiva implica aprender a confiar, escucharse el uno al otro, contagiarse, apasionarse, recordar, hacer memoria, reivindicar, caminar, respirar y perdonar. Como si fuera un código de ética, poco a poco, se es consiente de la relación con los otros, humanos y no humanos, se va comprendiendo la necesidad de despojarse de los deseos individuales para acercarse a los sueños, para decretar caminos posibles y hacerse poéticamente pragmáticos.

Ocho años de acciones colectivas permiten repensar los conceptos clásicos de familia, comunidad y movimiento social. La colectividad convoca experiencias, anécdotas, vivencias, risas, sonrisas, carcajadas y angustias extendidas, y permite actuar sin bandera, etiqueta, sin título, pero con aliados que fortalecen la acción. El éxito depende de las acciones invisibles 
de quienes ejercen la cooperación: animales, mascotas, familias, amigos e interesados que se anudan y permiten la dedicación continua al proceso, el relevamiento de tareas y liderazgos, y las tensiones y distensiones de largas jornadas de lecturas y audiencias.

La colectividad es poética: pensar ganar la batalla a multinacionales representaba más que una utopía. Lograr reducir 24000 ha de una licencia ambiental en cuatro años fue una juiciosa y dedicada tarea de reconocimiento territorial que demuestra el peso técnico y jurídico de los argumentos. La invitación es a crear poesía desde el territorio, con bases sólidas que evidencien los engaños de los proyectos extractivos, que nos ayuden a reconocer las gentes e instituciones con las que convivimos para vincularlas en la acción y aprender de sus estrategias. Son tantas y tantos los partícipes del proceso que no alcanzarían las líneas para describir cuán importante fue su momento y su acción, y cómo esta permitió congregar cerca de 2000 personas aquel noviembre de 2016 para decir que "en Boyacá no cambiamos ruana por overol petrolero", actuar consecuente con la negación de la modificación de la licencia en 2017 (Resolución 929/2017, de 8 de agosto; Resolución 00835/2018, de 5 de junio).

\section{CONCLUSIONES}

La colectividad es una estrategia de resistencia que evita el señalamiento de actores y garantiza el trabajo en red. Requiere un cambio de vida que implica reconocer y re-significar los paisajes, valorar lo que se tiene y gestionar procesos que permitan reivindicar las miradas, los saberes, las prácticas y las formas de hacer. Pensar y actuar en conjunto parece complejo por los intereses individuales de cada ser; sin embargo, cuando se es consciente de lo común y fundamental para un grupo humano, el camino se amplía y las luchas se dan. Se convierte en un fenómeno dinámico, propio de lo indeterminado y de lo inesperado por los cambios que pueda enfrentar o generar.

Lo colectivo no tiene receta, sino que depende de cada contexto, de las realidades, de las circunstancias y de los otros. Cada grupo humano reacciona de acuerdo con las intuiciones, con sus propios recursos y métodos, generan diálogos o silencios con las instituciones, regulan las acciones, confrontan y apelan. Así es como cada proceso vincula metodologías propias que dependen de las disciplinas, de los saberes y de las formas de hacer de sus actores. Para el Colectivo por la Protección de la Provincia de Sugamuxi, fue fundamental el retorno de quienes habían emigrado del departamento en busca de oportunidades académicas y laborales. Artistas, antropólogos, abogados, docentes, ingenieros, ecologistas, economistas y artesanos entendieron que desde los lenguajes comunes y las sensibilidades era posible concientizar a la población sobre las consecuencias ambientales de los proyectos extractivos.

La fotografía, el audio, el video y la georreferenciación fueron herramientas claves en los procesos de documentación; los habitantes reconocieron que no se necesita tener la cámara profesional para documentar los problemas, sino que con el celular es posible tener registros adecuados que funcionan como evidencias técnicas y jurídicas, con los cuales alimentar los procesos de resistencia en los territorios. A su vez, la pintura, el dibujo y la didáctica permitieron triangular la información documentada en medios audiovisuales y traducirla a lenguajes más cotidianos y sensibles en las calles, las escuelas y los escenarios comunitarios. 
Por fortuna, el reconocimiento territorial y su documentación posibilitaron escenarios de reflexión, discusión y recreación de las realidades. Los procesos formativos permitieron empoderar las comunidades con dispositivos que activaron y conectaron la memoria. Como un ejercicio de memoria biocultural, el Colectivo por la Protección de la Provincia de Sugamuxi dio ese salto académico del hacer, salió de los discursos y la teoría para comprender los saberes y las formas de hacer, entendió que no hay verdades sino interpretaciones, que no es ni negro ni blanco, que la realidad es diversa por naturaleza y que desde ahí es que se construye. La lección en el proceso es lograr combinar los colores, ponerlos en diálogo y crear desde sus posibilidades nuevas realidades.

\section{NOTAS}

1. Documento ICANH 130 1443, 8 de abril de 2014.

\section{REFERENCIAS}

Acosta, Myriam. 2015. "En la protección de la laguna de Tota y sus municipios aledaños". Consultado: 20 de abril de 2018. http://www.semillas.org.co/es/en-la-protecci

Caracol Radio. 2016. "Comunidad se opone a intervención petrolera en Pesca, Boyacá". Consultado: 11 de mayo de 2018. https://caracol.com.co/emisora/2016/11/08/tunja/1478616649_926835.html.

Colectivo por la Protección de la Provincia de Sugamuxi. 2013. "Modificaciones de licencia ambiental proyectos Muisca y Buenavista". Consultado: 5 de agosto de 2018. https://sites.google.com/site/ colectivosugamuxi/petroleo-provincia-de-sugamuxi/modificaciones-de-licencia-ambiental-proyectosmuisca-y-buenavista

- 2014. "Bloque MNorte a cargo de Maurel and Prom". Consultado: 3 de mayo de 2018. https://sites. google.com/site/colectivosugamuxi/boyaca-con-ruana-y-sombrero-protege-su-suelo/historial-demaurel-and-prom

- 2016. "Todos los videos". Consultado: 20 de noviembre de 2018. https://www.facebook.com/ pg/Colectivo-por-la-Protecci\% C3 \% B3n-de-la-Provincia-de-Sugamuxi-354171434628733/ videos/?ref=page_internal

- 2018. "Bloque Buena Vista a cargo de Unión Temporal Omega Energy". Consultado: 3 de agosto de 2018. https://sites.google.com/site/colectivosugamuxi/boyaca-con-ruana-y-sombrero-protege-susuelo/mineria-en-paipa

— 2019. "Qui est Getulio Montaña Laguna". Consultado: 18 de mayo de 2019. https://sites.google.com/ site/colectivosugamuxi/home/qui-est-getulio-montana-laguna

Contagio Radio. 2016. "210 cuerpos de agua cerca al lago de Tota desaparecieron por actividad petrolera". Consultado: 9 de noviembre de 2018. https://www.rds.org.co/es/novedades/210-cuerpos-de-aguacerca-al-lago-de-tota-desaparecieron-por-actividad-petrolera

Contraloría General de la República. 2013. "Actuación especial: evaluación de la gestión del lago de Tota". Consultado: 10 de diciembre de 2018. https://app.box.com/s/jitx0paswm660p1 rqwbt

Corpoboyacá (Corporación Autónoma Regional de Boyacá). 2006. Plan de ordenación y manejo ambiental de la cuenca alta del río Chicamocha. Tunja: Corpoboyacá. 
DNP (Departamento Nacional de Planeación). 2014. "Plan Nacional de Desarrollo 2014-2018: Todos por un nuevo país". Consultado: 6 de mayo de 2018. https://www.minagricultura.gov.co/planeacion-controlgestion/Gestin/Plan \%20de \%20Acci \%C3 \%B3n/PLAN \%20NACIONAL \%20DE \%20DESARROLLO \%202014 \%20- \%202018 \%20TODOS \%20POR \%20UN \%20NUEVO \%20PAIS.pdf

Foster, George. 1980. Las culturas tradicionales y los cambios técnicos. México: Fondo de Cultura Económica.

Getulio Muisca. 2012. "Grave denuncia sobre actividades de exploración sísmica terrestre en Boyacá". Consultado: 10 de octubre de 2018. https://www.youtube.com/watch?v=iA2355wq1YE

- 2012a. "Impactos de la petrolera Maurel \& Prom en Boyacá ¿Quién responde?". Consultado: 10 de octubre de 2018. https://www.youtube.com/watch?v=T-4PrBu_b8A

— 2013. “ ¿Petróleo en Boyacá? Desarrollo arrollador". Consultado: 12 de agosto de 2018. https://www. youtube.com/watch?v=WyYJSHARFy8

— 2014. "Omega Energy: ejemplos del impacto de la industria petrolera en Boyacá". Consultado: 12 de septiembre de 2018. https://www.youtube.com/watch?v=g5c4wUe0xhw

IGAC (Instituto Geográfico Agustín Codazzi). 2014. “El 43 por ciento del suelo de Boyacá está sobreutilizado; se estudiarán 103.900 hectáreas de humedales y páramos." Consultado: 3 de mayo de 2018. https:// noticias.igac.gov.co/es/contenido/el-43-por-ciento-del-suelo-de-boyaca-esta-sobreutilizado-seestudiaran-103900-hectareas-de

López Estupiñán, Laura. 2015. “Papas y tierras en Boyacá: investigación etnobotánica y etnohistórica de uno de los principales productos de la alimentación colombiana". Boletín de Antropología Universidad de Antioquia 30:170-1

Ministerio del Ambiente. 1997. "Guía básica ambiental para programas de exploración sísmica terrestre". Consultado: 3 de febrero de 2017. https://acp.com.co/web2017/es/guias/141-guia-ambientalexploracion-sismica-1998/file

Mujer Rural y Derecho a la Tierra. 2018. “Cómic para la divulgación Getulio Montaña Laguna, Quejulia Páramo Flores, Carmina Mar y Cubio Montaña Piedra (Colombia)." Consultado: 10 de mayo de 2018. https://www.landcoalition.org/sites/default/files/documents/resources/comunicacion_ getulioyquejulia_colombia.pdf

Pineda, Felipe. 2014. "Boyacá: entre el carbón y la resistencia civil". Las2Orillas. 16 de diciembre. Consultado: 12 de diciembre de 2017. http://www.las2orillas.co/boyaca-carbon-resistencia-civil/

Procuraduría General de la Nación. 2012. "Concepto técnico jurídico proyecto: Área de interés exploratorio Muisca hoy MNorte". Consultado: 3 de julio de 2018. https://es.scribd.com/document/131932751/ Concepto-Tecnico-juridico-Procuraduria-MNorte

RCN Radio. 2013. "Se socializó en audiencia pública, solicitud de ampliación de licencia a Omega Energy". Consultado: 20 de mayo de 2018. https://www.rcnradio.com/colombia/se-socializo-en-audienciapublica-solicitud-de-ampliacion-de-licencia-omega-energy-77361

Resolución 0376/2014, de 11 de abril, por la cual se niega la modificación de la licencia ambiental global otorgada mediante Resolución 1156 del 27 de junio de 2007, y se dictan otras disposiciones.

Resolución 0867/2015, de 24 de julio, por la cual se modifica la Resolución 1156 del 27 de junio de 2007 y se toman otras determinaciones.

Resolución 929/2017, de 8 de agosto, por la cual se otorga una licencia ambiental y se toman otras determinaciones.

Resolución 00835/2018, de 5 de junio, por la cual se resuelve el recurso de reposición interpuesto contra la Resolución 929 del 8 de agosto de 2017.

Reyes Becerra, Carmenza. 2014. "Boyacá pionera en la legalización de predios". Consultado: 2 de mayo de 2018. http://www.boyaca.gov.co/prensa-publicaciones/noticias/2642-boyac \%C3 \%A1-pioneraen-legalizaci \%C3 \%B3n-de-predios

Rodríguez Cuenca, José Vicente. 2003. Dientes y diversidad humana: avances de la antropología dental. Bogotá: Universidad Nacional de Colombia.

Semana Sostenible. 2016. "El proyecto petrolero que 'incendiaría' a Boyacá." Consultado: 23 de agosto de 2018. https://sostenibilidad.semana.com/medio-ambiente/articulo/sogamoso-y-el-proyectopetrolero-que-incendiaria-a-boyaca/36724 